\title{
Changes in salivary oxytocin after inhalation of clary sage essential oil scent in term-pregnant women: a feasibility pilot study
}

\author{
Yuriko Tadokoro ${ }^{{ }^{*}}$ (D), Shigeko Horiuchi ${ }^{2,3}$ (D), Kaori Takahata ${ }^{1}$, Takuya Shuo ${ }^{4}$, Erika Sawano ${ }^{5}$ \\ and Kazuyuki Shinohara ${ }^{5}$
}

\begin{abstract}
Objectives: This pilot study using a quasi-experimental design was conducted to evaluate the feasibility (i.e., limited efficacy, practicality, and acceptability) of our intervention protocol involving inhalation of the scent of clary sage essential oil by pregnant women and measurement of their preinhalation and postinhalation oxytocin levels.

Results: Participants were women of singleton pregnancies between 38 and 40 gestation weeks $(N=11)$. The experiment group $(n=5)$ inhaled the scent of clary sage essential oil diluted 50-fold with $10 \mathrm{~mL}$ of odorless propylene glycol for $20 \mathrm{~min}$. Regarding limited efficacy, the oxytocin level $15 \mathrm{~min}$ postinhalation increased in 3 women and was unmeasurable in 2 . The control group $(n=6)$ inhaled similarly without the 50 -fold dilution of clary sage essential oil. Their oxytocin level increased in 2 women, decreased in 2, and was unmeasurable in 2. Uterine contraction was not observed in both groups. Regarding practicality, 3 of the 11 women could not collect sufficient saliva. The cortisol level decreased in both groups postinhalation. The protocol had no negative effects. Regarding acceptability, burden of the protocol was not observed.

Trial registration The Clinical Trials Registry of University Hospital Medical Information Network in JapanUMIN000017830. Registered: June 8, 2015
\end{abstract}

Keywords: Pregnant women, Induction of labor, Complementary and alternative medicine, Aromatherapy, Clary sage essential oil, Inhalation, Salivary oxytocin, Uterine contraction, Salivary cortisol, Feasibility study

\section{Introduction}

Medical induction of labor has been widely used [1-3]. However, it occasionally interferes with physiologic childbirth, necessitating the evaluation of the effects of complementary and alternative medicine for stimulating labor $[4,5]$. Aromatherapy has been complementarily used for stimulating and strengthening labor contraction (e.g., repeated use of the scent of clary sage essential oil) [6-10]. However, there are few studies that

\footnotetext{
*Correspondence: 14dn011@slcn.ac.jp

1 St. Luke's International University, 10-1 Akashicho, Chuo, Tokyo 104-0044, Japan

Full list of author information is available at the end of the article
}

have investigated the effects of aromatherapy on labor stimulation.

Labor involves uterine contractions (UCs) caused by oxytocin [11]. Clary sage essential oil is thought to stimulate labor by increasing the oxytocin level. However, studies evaluating changes in the oxytocin level of pregnant women by any intervention remain scarce. Thus, we conducted this feasibility study as a basis for future larger studies $[12,13]$.

This research was a pilot study to determine the feasibility of our intervention protocol in which pregnant women inhaled the scent of clary sage essential oil. Our primary objective was to evaluate the protocol's limited efficacy in terms of the oxytocin level and UC. Our 
secondary objective was to evaluate the protocol's practicality (i.e., saliva collection ability, cortisol level, and negative effects on participants) and acceptability (i.e., burden of intervention and scent perception).

\section{Main text \\ Methods}

This research was a feasibility pilot study conducted using a quasi-experimental design with two arms: experiment group and control group.

\section{Participants and setting}

The inclusion criteria included low-risk pregnant women between 38 and 40 gestation weeks before labor onset for planning spontaneous delivery. The exclusion criteria included age $(<25$ or $>35)$ and aromatherapy allergy. The Additional file 1 shows details of participants' criteria and request to participants until the intervention.

Sample size was not calculated because this pilot study aimed to provide a descriptive evaluation of the feasibility of the intervention protocol. Thus, about five participants for each group were considered appropriate. The first half of the participants was assigned to the control group and the last half to the experiment group. The feasibility pilot study was conducted at a birth center in Tokyo, Japan between June 2015 and August 2015.

The participants, experimental setting staff, and biologists measuring the outcomes were masked about the participant allocation.

\section{Intervention}

The inhalation intervention for each participant was started at 13:00. The participants rinsed their mouth with water, answered a questionnaire, and drank $100 \mathrm{~mL}$ of water. After $10 \mathrm{~min}$ from the water intake, the first saliva sample was collected [14] and the inhalation was started.

The experiment group inhaled the scent of clay sage essential oil (Salvia sclarea, Tree of life, Tokyo, Japan) [15] for $20 \mathrm{~min}$. The scent was produced by bubbling using an air pump at $2.0 \mathrm{~mL} / \mathrm{min}$ in an airtight bottle containing $10 \mathrm{~mL}$ of odorless propylene glycol diluted with 50-fold of clary sage essential oil and delivered $10 \mathrm{~cm}$ away from the nostril [16]. The Additional file 2 provides details of the inhalation intervention.

The control group inhaled similarly using the same device but without the dilution of clary sage essential oil with $10 \mathrm{~mL}$ of propylene glycol.

\section{Outcome measures}

For the primary objective, salivary oxytocin level was measured at four time points: $10 \mathrm{~min}$ preinhalation (baseline), and 15, 30, and 60 min postinhalation [17-21]. At each measurement point, at least $1.0 \mathrm{~mL}$ of saliva was collected in a polypropylene tube by passive drool after pooling saliva in the mouth for $3 \mathrm{~min}[22,23]$. When the volume was less than $1.0 \mathrm{~mL}$, saliva was recollected as in the first attempt. The participants drank $100 \mathrm{~mL}$ of water again immediately after saliva collection at $30 \mathrm{~min}$ postinhalation. Salivary samples were frozen in a box containing dry ice after each collection point, and were then stored at $-80{ }^{\circ} \mathrm{C}$ following completion of each intervention.

Oxytocin level was assayed in duplicates by enzymelinked immunosorbent assay (ELISA; ENZO Life Sciences, NY, USA) following the protocol of Carter et al., but with the addition of $500 \mathrm{KIU} / \mu \mathrm{L}$ aprotinin $[22,24-$ $26]$. The inter-assay and intra-assay coefficients of variability were reported to be $12.6-13.3$ and $11.8-20.9 \%$, respectively [27].

Objective UCs were monitored by cardiotocography. The frequency of subjective UCs was asked from the participants at preinhalation, and at 30 and $60 \mathrm{~min}$ postinhalation.

For the secondary objective, the volume of the collected saliva and the number of attempts to collect saliva, both of which constitute saliva collection ability, were recorded. To evaluate whether our intervention protocol can be conducted without causing stress [28], the salivary cortisol level was measured at the same four time points of oxytocin level measurement. Saliva was collected in a separate tube to measure oxytocin and cortisol levels at each measurement point. The assay was conducted by ELISA in duplicates (Salimetrics, PA, USA) following the manufacturer's instructions.

Negative effects included an abnormal fetal heart rate pattern during the intervention as monitored by cardiotocography, and neonatal outcomes (Apgar score $<7$ and neonatal intensive care unit admission) were collected from the medical records. For the assessment of acceptability, the burden of intervention (inhalation, saliva and buccal mucosa collection, and participation) in both groups and the scent perception (preference and strength) in the experiment group were clarified at the end of the intervention.

Some basic characteristics which were reportedly related to a lower oxytocin level were identified [2934]. The Additional file 3 shows questionnaires and oral questions.

\section{Data analysis}

Outcomes were descriptively analyzed. The changes between the preinhalation and postinhalation oxytocin and cortisol levels and UC were compared between groups. Additionally, changes in oxytocin levels were statistically compared within groups by the Friedman test and between groups by the Mann-Whitney $U$ test with 
a two-sided 5\% level of significance using SPSS version 25.0J for Windows.

\section{Results}

Eleven women received the intervention: $5(\mathrm{E} 1-5)$ in the experiment group and $6(\mathrm{C} 1-6)$ in the control group. The Additional file 4 shows the participants' flow chart. Most of the participants' characteristics were similar between the groups (Table 1).

\section{Primary outcomes: limited efficacy}

The oxytocin level could be measured at all the measurement points in 3 women in the experiment group (Fig. 1 E1-3). Their oxytocin levels increased at $15 \mathrm{~min}$ and then decreased at $30 \mathrm{~min}$ postinhalation. The oxytocin level could be measured at all the measurement points in 4 women in the control group (Fig. 1 C1-4). The oxytocin level at 15 min postinhalation increased in 2 women and decreased in 2 women. However, there were no significant differences in the oxytocin level changes within groups and between groups. Postinhalation objective and subjective UCs were not observed in both groups.

\section{Secondary outcomes: practicality}

For saliva collection, 3 of the 11 women (27.2\%) failed to collect $1.0 \mathrm{~mL}$ of saliva even with 4 attempts. The other women could collect $1.0 \mathrm{~mL}$ or more of saliva within 3 attempts.
For oxytocin level measurement, 44 saliva samples were collected in both groups. Of the 44 samples, 9 contained $1.5 \mathrm{~mL}$ or more of saliva and the oxytocin level could be measured in all of them. Of the 44 saliva samples, 35 contained less than $1.5 \mathrm{~mL}$ of saliva and the oxytocin level could not be measured in 10 of these 35 samples $(28.5 \%)$ owing to the small saliva volume postcentrifugation.

The cortisol level at all the measurement points could be measured in 3 women in the experiment group (Fig. 2 E1, E2, E4). All their cortisol levels decreased at $15 \mathrm{~min}$ postinhalation. All the postinhalation cortisol levels remained lower than baseline, except in E2. In the control group, the cortisol level at all the measurement points could be measured in all the women (Fig. 2 C1-6). The cortisol levels at all the postinhalation measurement points were lower than baseline, except in C5.

Any negative effects on the fetal heart rate pattern during the intervention and on neonatal outcome were not observed.

\section{Secondary outcomes: acceptability}

All the burdens of the intervention were judged as moderate or light in both groups. All the women in the experiment group liked the scent of clary sage essential oil. The strength of the scent was judged as appropriate in E1 and E3, and as weak in E2, E4, and E5 in which the oxytocin level could be measured only in E2. The postinhalation oxytocin level in E2 was higher than the baseline.

Table 1 Basic characteristics of participants

\begin{tabular}{llll}
\hline & Experiment group $(\mathbf{n}=\mathbf{5})$ & Control group $(\mathbf{n}=\mathbf{6})$ & $\mathbf{p}$ value \\
\hline Age [SD] (years) & $31.4[2.7]$ & $32.6[1.8]$ & 0.429 \\
Gestation weeks [SD] & $38.2[0.2]$ & $38.2[0.1]$ & 0.662 \\
BMI [SD] & $23.4[3.5]$ & $24.6[2.5]$ & 0.662 \\
Education $\geq 12$ years & 5 & 5 & 1.000 \\
Married & 5 & 6 & - \\
Having children & 1 & 2 & 1.000 \\
Oxytocin receptor polymorphisms & & & 1.000 \\
rs 53576 (GG) & 0 & 1 & 0.567 \\
rs2254298 (GG) & 3 & 0 & 0.455 \\
rs1042778 (TT) & 1 & 0 & - \\
Depression (CES-D $\geq 16)$ & 0 & $30.0[3.1]$ & 0.126 \\
Anxiety & & $32.6[7.4]$ & 0.177 \\
A-Trait [SD] & $31.0[3.3]$ & $26.6[3.4]$ & \\
A-State [SD] & & & \\
\hline
\end{tabular}

Oxytocin receptor single nucleotide polymorphism was assayed using buccal mucosa samples collected after completing all saliva collections (TaqMan SNP genotyping assays, Applied Biosystems, Thermo Fisher, MA, USA)

The characteristics reportedly related to a lower oxytocin level were oxytocin receptor polymorphism (GG in rs53576 and rs2254298, and TT in rs1042778) [31-33]; having depression [29, 30,32], anxiety [33], a high body mass index and no children, and being married [34]. Depression and anxiety were assessed using the Japanese version of CES-D [35, 36] and STAI [37, 38]

SD standard deviation, BMI Body mass index, CES-D Center for Epidemiologic Studies Depression Scale, $A$-trait Trait anxiety of State-Trait Anxiety Inventory, A-State state anxiety of State-Trait Anxiety Inventory

p-value Mann-Whitney $U$ test or Fisher's exact test for comparisons between groups 

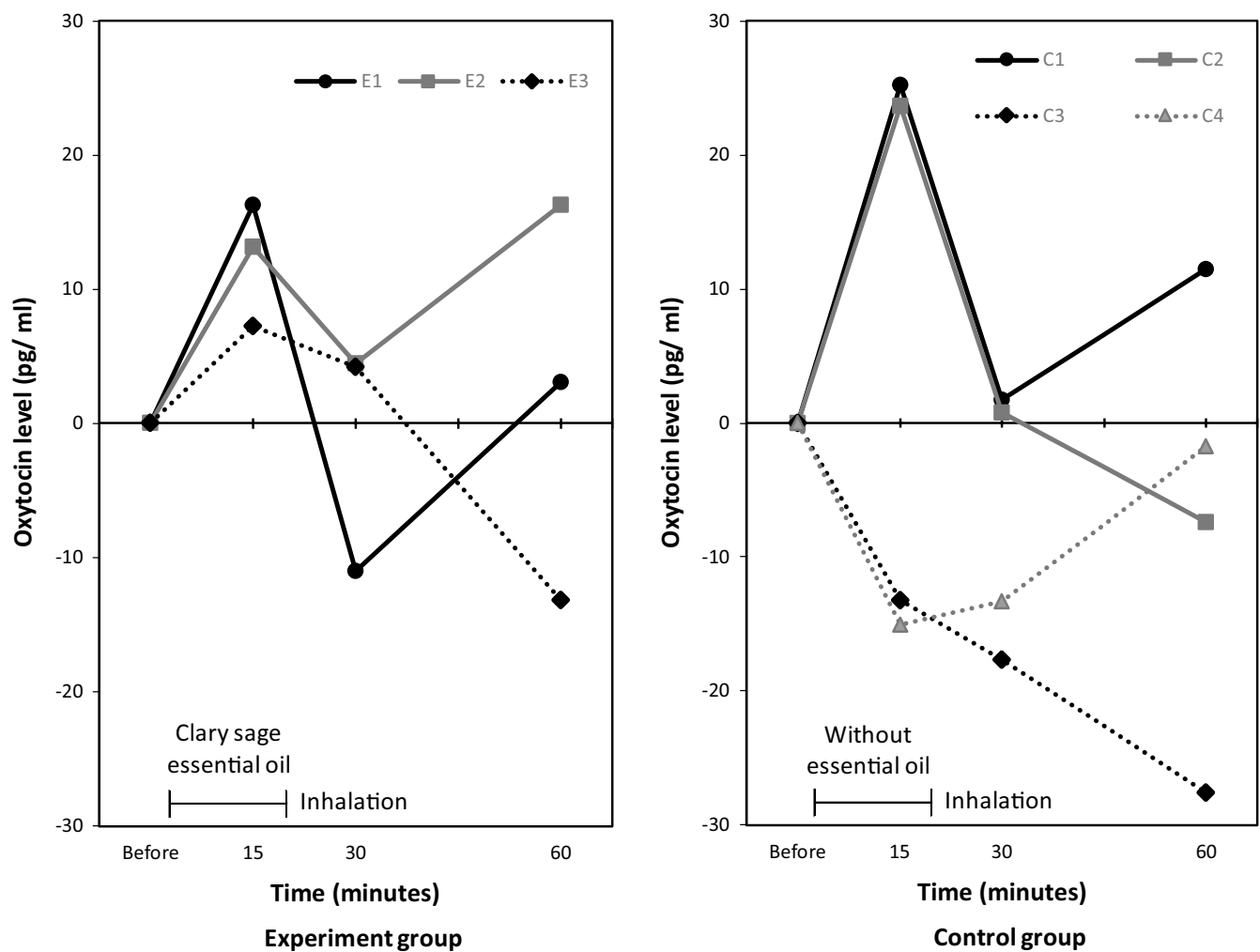

Fig. 1 Changes in preinhalation and postinhalation oxytocin levels. Left: Experiment group, $n=5, E 4$ and E5 are not shown because the oxytocin levels at all the time points were unmeasurable; Right: Control group, $n=6, C 5$ and C 6 are not shown because the oxytocin level at all the time points were unmeasurable. Comparison within groups, no significant differences in the oxytocin levels were found between preinhalation and postinhalation (experiment group: $p=0.241$; control group: $p=0.682$ ). Comparison between groups, no significant differences were found in the preinhalation levels of oxytocin $(p=0.310)$ and the changes between preinhalation and $15 \mathrm{~min}(p=1.000), 30 \mathrm{~min}(p=0.190)$, and $60 \mathrm{~min}$ $(p=0.690)$ postinhalation

\section{Discussion}

\section{Limited efficacy: oxytocin level and uterine contraction}

Inhalation of the scent of clary sage essential oil induced an increasing trend in the oxytocin level at $15 \mathrm{~min}$ postinhalation which was measured during the inhalation. This increase, however, could not be maintained at $30 \mathrm{~min}$ postinhalation measured at $10 \mathrm{~min}$ after finishing the inhalation. Thus, future studies should measure the oxytocin level at $15 \mathrm{~min}$ postinhalation and during or immediately after the inhalation of the scent of clary sage essential oil to evaluate the effects.

Uterine contraction was expected to be induced in accordance with the increase in the oxytocin level. However, postinhalation subjective and objective UCs were not observed in the experiment group. Regarding oxytocin receptors, their amount increases around the onset of labor [39] and their polymorphism is related the duration of labor and delivery [40]. Therefore, UC is affected by the oxytocin level as well as the amount and polymorphism of oxytocin receptors. Another possibility is that the increase in the oxytocin level might be small to cause UCs. Clary sage essential oil contains sclareol and is expected to have estrogen-like effects because sclareol has a structure similar to estrogen [41]. As estrogen enhances the release of oxytocin [34], it can be expected that a greater absorption of sclareol into the body would mean a larger increase in the oxytocin level. The present study used inhalation as an absorption route of the essential oil into the body, although skin absorption is also used in aromatherapy. In future studies, administration methods that facilitate the simultaneous skin and inhalation absorption of essential oils such as using a footbath should be adopted for cumulative effects on oxytocin release.

\section{Practicality: saliva collection ability, cortisol level, and negative effects}

In all the samples with $1.5 \mathrm{~mL}$ or more of saliva, the oxytocin level could be measured. Notably, $27.2 \%$ of women failed to collect $1.0 \mathrm{~mL}$ of saliva even with 4 attempts. The oxytocin level measurement can be further improved by collecting a minimum amount of $1.5 \mathrm{~mL}$ of saliva. 

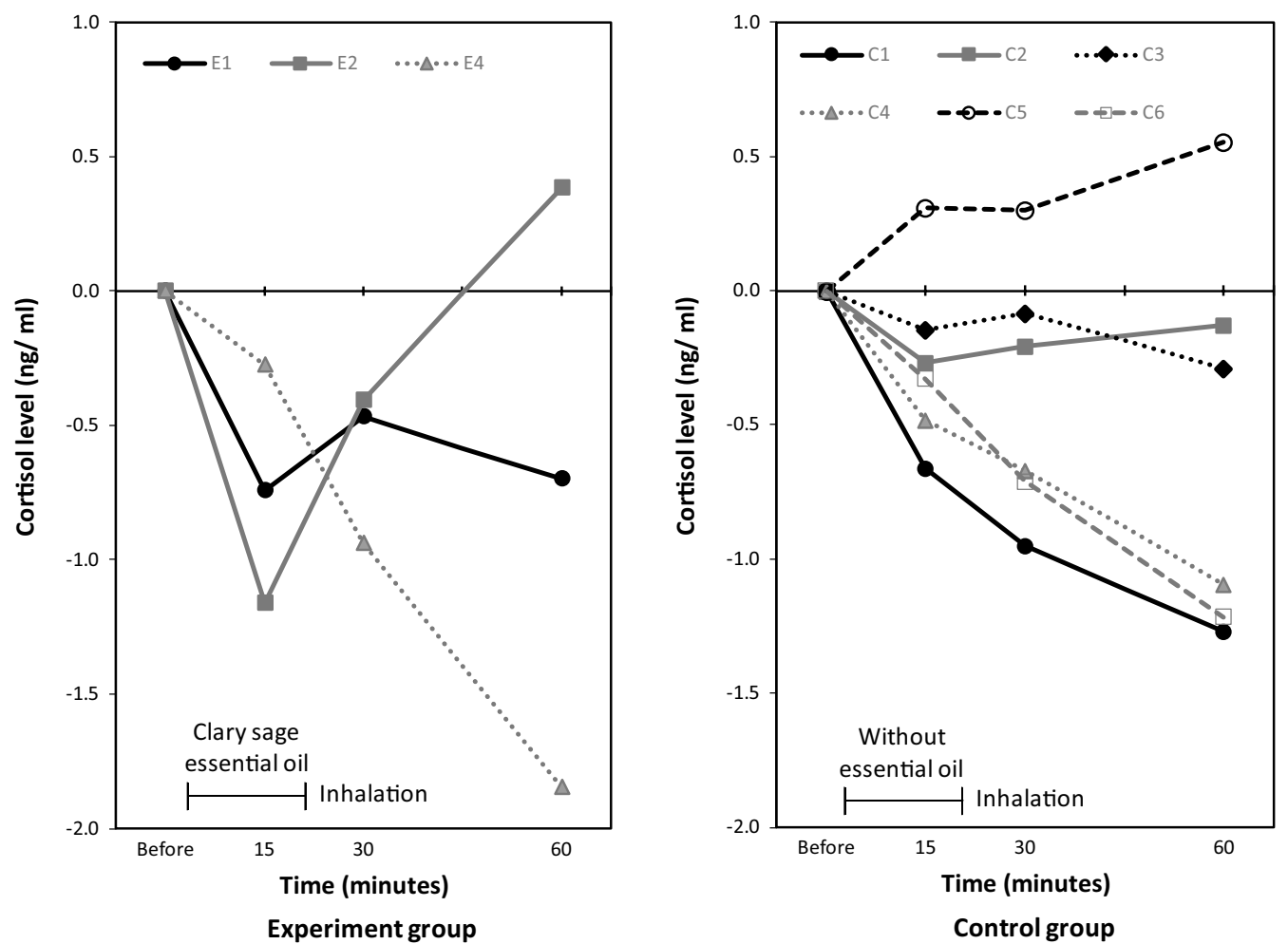

Fig. 2 Changes in preinhalation and postinhalation cortisol levels. Left: Experiment group, $n=5, E 3$ and E5 are not shown because the cortisol levels at all the time points were unmeasurable. Right: Control group, $n=6$

Moreover, the $27.2 \%$ of women with deficient saliva sample should be estimated in the sample size analysis. To obtain adequate saliva volumes, any stimuli that promote salivation consistently at each saliva collection may serve as a solution [14, 42]. Notably, studies indicating the required saliva volumes and deficiencies in the appropriate saliva volume for oxytocin level measurement remain scarce. Recently, the number of studies on oxytocin has been increasing, and novel findings can also be useful for other oxytocin studies.

The cortisol level showed a decreasing trend during the inhalation intervention in both groups, indicating the absence of intervention-induced stress. The intervention protocol had no negative effects.

\section{Acceptability: burden of intervention and scent perception}

The intervention protocol was within the acceptance level of the participants. Although 3 of 5 women judged the scent as weak, the oxytocin level increased postinhalation. In future studies, the same strength or a stronger scent can be considered.

\section{Conclusions}

This feasibility pilot study showed that inhalation of the scent of clary sage essential oil induced an increasing trend in the oxytocin level, but had no effect on UC. The intervention protocol showed good acceptability. In terms of practicality, it can be further adjusted to enable the collection of a sufficient amount of saliva for optimal measurement of the oxytocin level.

\section{Limitations}

- The limited efficacy of the inhalation could not be clearly confirmed owing to some missing oxytocin values.

- The method of oxytocin measurement did not include an extraction step, which has bearing on the reliability of the measurements. Future studies should consider the inclusion of an extraction step.

- The experiment group could easily guess the presence of the scent of clary sage essential oil which they liked. Thus, an increase in the oxytocin level from the perception of any preferred scent is possible. 


\section{Additional files}

Additional file 1. Participants' criteria and request to participants until intervention. Details of the inclusion and exclusion criteria of the participants and requests to the participants to avoid any possible disturbance of the oxytocin and cortisol measurement by enzyme immunosorbent assay until the intervention.

Additional file 2. Inhalation intervention. Details of inhalation intervention

Additional file 3. Questionnaires and oral questions. Preinhalation and postinhalation questionnaires and postinhalation oral questions translated from Japanese to English.

Additional file 4. Participants'flow chart. Flow chart of the participants from recruitment to analysis of outcomes.

\section{Abbreviations}

ELISA: enzyme-linked immunosorbent assay; UC: uterine contraction.

\section{Authors' contributions}

YT and SH designed the study, analyzed the data, and drafted the manuscript. YT conducted the experiments and collected the data. YT, KT, and TS organized the inhalation and saliva collection methods. TS analyzed the oxytocin receptor polymorphisms. ES and KS arranged the measurements of the salivary oxytocin and cortisol levels. KT, TS, ES, and KS critically reviewed and revised the draft of the study plan and the manuscript. All authors read and approved the final manuscript.

\section{Author details}

${ }^{1}$ St. Luke's International University, 10-1 Akashicho, Chuo, Tokyo 104-0044, Japan. ${ }^{2}$ Graduate School of Nursing Science, St. Luke's International University, 10-1 Akashicho, Chuo, Tokyo 104-0044, Japan. ${ }^{3}$ St. Luke's Maternity Care Home, 24 Akashicho, Chuo, Tokyo 104-0044, Japan. ${ }^{4}$ Hokuriku University, 3 Ho, Kanagawamachi, Kanazawa, Ishikawa 920-1181, Japan. ${ }^{5}$ Graduate School of Biomedical Sciences, Nagasaki University, 1-12-4 Sakamoto, Nagasaki 852-8523, Japan.

\section{Acknowledgements}

We are grateful to all the participants, the staff at the data collection setting, and Ms. Kanae Kitagawa (aromatherapist and aromatherapy instructor) for their professional assistance and advice. We are indebted to Dr. Edward F. Barroga (http://orcid.org/0000-0002-8920-2607) for his editorial review of our manuscript. Parts of this study were presented in a poster presentation session at the 19th East Asian Forum of Nursing Scholars, Chiba, Japan in 2016 and at the 57th Annual Meeting of the Japan Society of Maternal Health, Tokyo, Japan. Our study closely adhered to the CONSORT guidelines.

\section{Competing interests}

The authors declare that they have no competing interests.

\section{Availability of data and materials}

All data generated or analysed during this study are included in this published article.

\section{Consent for publication}

All participants provided written informed consent to publish this study.

\section{Ethics approval and consent to participate}

This study was approved by the Research Ethics Committee of St. Luke's International University (No. 15-04). The participants provided written informed consent to participate in the study. This study was registered in the Clinical Trials Registry of University Hospital Medical Information Network in Japan (UMIN000017830).

\section{Funding}

This study was financially supported by MEXT/JSPS KAKENHI B (26293475) and A (17H01613), the Society for Women's Health Science Research, and Yamaji Fumiko Nursing Research Fund. These funding sources were not involved in the design, intervention, data collection and analysis, and publication of this study.

\section{Publisher's Note}

Springer Nature remains neutral with regard to jurisdictional claims in published maps and institutional affiliations.

Received: 4 September 2017 Accepted: 30 November 2017

Published online: 08 December 2017

\section{References}

1. ACOG Committee on Practice Bulletins-Obstetrics. ACOG practice Bulletin No. 107: induction of labor. Obstet Gynecol USA. 2009;114:386-97.

2. Osterman MJK, Martin JA. Data brief 155: recent declines in induction of labor by gestational age. NCHS Data Brief. 2014;155:1-8.

3. Terada M, Matsuda Y, Ogawa M, Matsui H, Satoh S. Effects of maternal factors on birth weight in Japan. J Pregnancy. 2013;2013:172395.

4. Mayberry LJ, Avery MD, Budin W, Perry S. Improving maternal and infant outcomes by promoting normal physiologic birth on hospital birthing units. Nurs Outlook. 2017:65:240-1.

5. American College of Nurse-Midwives, Midwives Alliance of North America, National Association of Certified Professional Midwives. Supporting healthy and normal physiologic childbirth: a consensus statement by the American College of Nurse-Midwives, Midwives Alliance of North America, and the National Association of Certified Professional Midwives. J Midwifery Womens Health. 2012;57:529-32.

6. Musil A. Labor encouragement with essential oils. Midwifery Today Int. Midwife. 2013;107:57-8.

7. Burns EE, Blamey C, Ersser SJ, Barnetson L, Lloyd AJ. An investigation into the use of aromatherapy in intrapartum midwifery practice. J Altern Complement Med. 2000;6:141-7.

8. Fritz S. Essential oils for pregnancy, birth and babies. Kindle 2nd. Arizona: Gently Born Publications; 2015.

9. Stadelmann I. Original stadelmann aroma blends: essential oils for living, giving birth, dying. Kindle 1st. Wiggensbach: Stadelmann publishing; 2013. p. 34

10. Omoto C. Boseikangoto aromatherapy [Maternal Nursing and Aromatherapy]. In: Nursing Research in Japanese Society of Aromatherapy, editor. Nursenotameno Aromatherapy. [Aromatherapy for Nurse]. 1st ed. Osaka: Medicus Shuppan Publishers Co., Ltd.; 2005. p. 133-44.

11. Kamel RM. The onset of human parturition. Arch Gynecol Obstet Springer Verlag. 2010;281:975-82.

12. Eldridge SM, Lancaster GA, Campbell MJ, Thabane L, Hopewell S, Coleman $C L$, et al. Defining feasibility and pilot studies in preparation for randomised controlled trials: development of a conceptual framework. PLOS ONE. 2016;11:e0150205.

13. Bowen DJ, Kreuter M, Spring B, Cofta-Woerpel L, Linnan L, Weiner D, et al. How we design feasibility studies. Am J Prev Med. 2009;36:452-7.

14. Salimetrics LCC. Saliva Collection and Handling Advice, 3rd ed; 2015. https://www.salimetrics.com/assets/documents/Saliva_Collection_Handbook.pdf.

15. Tree of Life Co. Ltd. Seibun bunsekihyo kurarise-ji, rotto No. 51 [Table of composition: Clary sage essential oil, Lot No. 51]. Tokyo, Japan; 2015.

16. Kuroda K, Inoue N, Ito Y, Kubota K, Sugimoto A, Kakuda T, et al. Sedative effects of the jasmine tea odor and (R)-(-)-linalool, one of its major odor components, on autonomic nerve activity and mood states. Eur J Appl Physiol. 2005;95:107-14.

17. Atsumi T, Tonosaki K. Smelling lavender and rosemary increases free radical scavenging activity and decreases cortisol level in saliva. Psychiatry Res. 2007:150:89-96.

18. Lee K-B, Cho E, Kang Y-S. Changes in 5-hydroxytryptamine and cortisol plasma levels in menopausal women after inhalation of clary sage oil. Phytother. Res. 2014;28:1599-605. 
19. Yamaguchi M, Tahara Y, Kosaka S. Influence of concentration of fragrances on salivary al pha-amylase. Int J Cosmet Sci. 2009;31:391-5.

20. Uvnäs-Moberg K. Oxytocin may mediate the benefits of positive social interaction and emotions. Psychoneuroendocrinology. 1998;23:819-35.

21. Seol GH, Lee YH, Kang P, You JH, Park M, Min SS. Randomized controlled trial for Salvia sclarea or Lavandula angustifolia: differential effects on blood pressure in female patients with urinary incontinence undergoing urodynamic examination. J Altern Complement Med. 2013;19:664-70.

22. Daughters K, Manstead ASR, Hubble K, Rees A, Thapar A, van Goozen SHM. Salivary oxytocin concentrations in males following intranasal administration of oxytocin: a double-blind, cross-over study. PLoS ONE. 2015;10:e0145104.

23. Goebel-Stengel M, Stengel A, Taché Y, Reeve JR. The importance of using the optimal plasticware and glassware in studies involving peptides. Anal Biochem. 2011:414:38-46.

24. McCullough ME, Churchland PS, Mendez AJ. Problems with measuring peripheral oxytocin: can the data on oxytocin and human behavior be trusted? Neurosci Biobehav Rev. 2013:37:1485-92.

25. Carter CS, Pournajafi-Nazarloo H, Kramer KM, Ziegler TE, White-Traut R, Bello D, et al. Oxytocin: behavioral associations and potential as a salivary biomarker. Ann NY Acad Sci. 2007;1098:312-22.

26. Niwayama R, Nishitani S, Takamura T, Shinohara K, Honda S, Miyamura T, et al. Oxytocin mediates a calming effect on postpartum mood in primiparous mothers. Breastfeed Med. 2017;12:103-9.

27. Enzo Life Sciences Inc. Product Manual Oxytocin ELISA kit; 2015.

28. Clements AD. Salivary cortisol measurement in developmental research: where do we go from here? Dev Psychobiol. 2013;55:205-20.

29. Holt-Lunstad J, Birmingham W, Light KC. The influence of depressive symptomatology and perceived stress on plasma and salivary oxytocin before, during and after a support enhancement intervention. Psychoneuroendocrinology. 2011;36:1249-56.

30. Cyranowski JM, Hofkens TL, Frank E, Seltman H, Cai H-M, Amico JA. Evidence of dysregulated peripheral oxytocin release among depressed women. Psychosom Med. 2008;70:967-75.

31. Feldman R, Zagoory-Sharon O, Weisman O, Schneiderman I, Gordon I, Maoz $\mathrm{R}$, et al. Sensitive parenting is associated with plasma oxytocin and polymorphisms in the OXTR and CD38 genes. Biol Psychiatry. 2012;72:175-81.
32. Skrundz M, Bolten M, Nast I, Hellhammer DH, Meinlschmidt G. Plasma oxytocin concentration during pregnancy is associated with development of postpartum depression. Neuropsychopharmacology. 2011;36:1886-93.

33. Heim C, Young LJ, Newport DJ, Mletzko T, Miller AH, Nemeroff CB. Lower CSF oxytocin concentrations in women with a history of childhood abuse. Mol. Psychiatry. 2009;14:954-8.

34. Uvnas-Moberg K, Petersson M. Oxytocin, a mediator of anti-stress, well-being, social interaction, growth and healing. Z Psychosom Med Psychother. 2005;51:57-80.

35. Shima S. CES-D Scale: Utsubyo (yokuutsujotai) jikohyoka syakudo [CES-D Scale: self-administered scale for depression (depressive state)], 1st edn. Chiba Test Cent. Tokyo: Chiba Test Center Co., Ltd.; 1998.

36. Radloff $L S$. The CES-D scale a self-report depression scale for research in the general population. Appl Psychol Meas Sage Publ. 1977;1:385-401.

37. Spielberger CD, Gorsuch RL, Lushene RE. STAI manual for the State-trait anxiety inventory ("self-evaluation questionnaire"). California: Consulting Psychologists Press; 1970.

38. Shimizu H, Imae K. Development of the Japanese edition of the Spielberger State-Trait Anxiety Inventory (STAl) for student use. Japanese J Educ Psychol. 1981;29:348-53.

39. Fuchs AR, Fuchs F, Husslein P, Soloff MS. Oxytocin receptors in the human uterus during pregnancy and parturition. Am J Obstet Gynecol. 1984;150:734-41.

40. Terkawi AS, Jackson WM, Thiet MP, Hansoti S, Tabassum R, Flood P. Oxytocin and catechol-O-methyltransferase receptor genotype predict the length of the first stage of labor. Am J Obstet Gynecol. 2012;207:184. e1-188.e1.

41. Tisserand R, Young R. Essential oil safety: a guide for health care professionals Kindle. 2nd ed. Edinburgh: Churchill Livingstone, Elsevier Health Sciences; 2013.

42. White-Traut R, Watanabe K, Pournajafi-Nazarloo H, Schwertz D, Bell A, Carter CS. Detection of salivary oxytocin levels in lactating women. Dev. Psychobiol. 2009;51:367-73.

\section{Submit your next manuscript to BioMed Central and we will help you at every step:}

- We accept pre-submission inquiries

- Our selector tool helps you to find the most relevant journal

- We provide round the clock customer support

- Convenient online submission

- Thorough peer review

- Inclusion in PubMed and all major indexing services

- Maximum visibility for your research

Submit your manuscript at www.biomedcentral.com/submit
O) BioMed Central 